\title{
¿Es posible una asistencia sanitaria mejor y un acortamiento marcado de las listas de espera?
}

\author{
Manuel Angel Seco Fernández \\ Médico internista jubilado del $\mathrm{CHOU}$
}

La respuesta a esta pregunta es: si, y mucho. Para ello se precisa un aumento del porcentaje del PIB para sanidad (que ha ido cayendo progresivamente hasta el actual $5.8 \%$, mientras que en EE.UU. se encuentra actualmente en un $17 \%$ y estiman llegar al 20\% en los próximos años). Pese a ello, y gracias al sistema MIR (1978) y medicina de familia (1983), hemos alcanzado el puesto número 4 en la clasificación por excelencia de la asistencia sanitaria en el mundo. Además de este aumento en el PIB es necesario un pacto de estado para evitar los vaivenes políticos, el mismo pacto que viene siendo reclamado para la mejora de la educación.

Para un septuagenario como yo, el peor invento de la democracia fue el café para todos recetado por los padres dela patria: 17 autonomías. Es decir, 17 ministerios de sanidad y desigualdad. Han echado abajo la Ley General de Sanidad de Ernest Lluch de cuyos cuatro principios (universalidad, equidad, eficiencia y libertad de elección) apenas se cumple el primero.

Una vez conseguidos los dos puntos anteriores (aumento del PIB y pacto de Estado) son muy necesarios ciertos cambios que fundamentalmente deberían tener lugar en medicina de familia, medicina hospitalaria y en la imbricación entre ambas medicinas. Y, por último, la transformación de los hospitales en una empresa eficiente dejando atrás la empresa ruinosa y dedocrática que es en la actualidad.

\section{Cambios en medicina de familia}

La tasa de médicos por 10.000 habitantes en este nivel es bastante inferior a la media europea y se calcula que se precisarán alrededor de 2.200 nuevos médicos de familia para adaptar el número de cartillas por médico, reducir la saturación de más de 40 consultas/día por facultativo y con ello aumentar el tiempo de atención a cada paciente. Por otra parte, el presupuesto de sanidad primaria es bajísimo actualmente; del $20 \%$ inicial se ha pasado a un $15 \%$ en la actualidad y continúa distanciándose del dedicado a la medicina hospitalaria. Se calcula que será necesario elevarlo hasta un 25\%. Pero además deberá darse más importancia al médico de familia, considerado la piedra angular del sistema sanitario, tanto en los centros de salud como en las facultades de medicina (formando parte del profesorado de familia) y en la investigación. Sus capacidades diagnósticas resolutivas se incrementarán al tener más medios y más tiempo para realizar cirugía menor, ecografía clínica, más peticiones de estudios diagnósticos sin pasar por el tamiz del médico especialista y más tiempo para realizar la detección y seguimiento del paciente crónico y/o frágil (siendo éste el problema más serio de la medicina actual por la longevidad creciente y la altísima tasa de ocupación de camas hospitalarias). En este apartado, el médico de familia, una vez haya identificado este tipo de pacientes y ayudado por personal de enfermería, asistentes sociales, fisioterapeutas... realizaría controles preventivos, revisiones cambios en el tratamiento... evitando así descompensaciones e ingresos hospitalarios.

Sugiero que los médicos de familia roten en torno a dos semanas cada cinco años en sus hospitales de referencia. Realizarían por las mañanas labor asistencial con internistas o especialistas que ellos elijan y por la tarde perfeccionarían sus habilidades 0 adquirirían otras nuevas con los servicios que consideren más idóneos. A la Comisión de Docencia le compete programar y coordinar estas rotaciones. Así, por un mecanismo de feed-back, se potenciarán las relaciones entre medicina primaria y hospitalaria, actualmente escasas. Estas rotaciones las he compartido durante mi formación como MIR en la Fundación Jiménez Díaz de Madrid.

\section{Cambios en medicina hospitalaria}

Durante mucho tiempo se ha considerado al hospital el único lugar adecuado para los ingresos de pacientes con enfermedades agudas, graves, crónicos, descompensados, realización de técnicas diagnósticas complejas y tratamientos complicados o cirugías mayores. Poco a poco ha venido siendo sustituido por la hospitalización a domicilio, hospital de día y cirugía ambulatoria. Aquí, en el hospital, es donde considero muy necesario el papel central del internista.

Cuando me preguntan qué es un internista suelo responder con humor: "un médico del interior con vistas". A continuación matizo lo que ya sabemos: un excelente explorador y diagnosticador, muy eficiente en la elaboración de historias clínicas y, sobre todo, con una visión integradora de todos y cada uno de los problemas del paciente y con un gran sentido del trabajo en equipo. En torno a un $20 \%$ de las altas hospitalarias son realizadas por internistas. Propongo aumentar el número de internistas hospitalarios para ocuparse de un porcentaje elevado de los enfermos de los distintos servicios de especialidades, enfermos, que 0 bien son crónicos o bien tienen pocas dificultades diagnósticas o terapeúticas y que, en todo caso, el internista puede resolver. De esta forma el especialista podrá dedicar más tiempo a realizar sus técnicas instrumentales con muchos más pacientes ambulantes 0 ingresados, reduciendo significativamente, de esta manera, la lista de espera. Propongo asimismo-reforzar o impulsar la figura del especialista consultor que en el horario habitual de mañana podrá ser requerido en cualquier lugar del hospital (hospitalización, consultas, áreas de urgencias, áreas de procedimientos diagnósticos....). Su labor de consultor será la de aconsejar, tanto al internista como a otros especialistas, en el diagnóstico y tratamiento de los pacientes en tiempo real. Estos dos rasgos, disponibilidad e inmediatez en horario de 
mañana todos los días, acortarían la estancia media de los pacientes notablemente.

En todos los centros de salud cuya población rondara los 50.000 habitantes se situaría un internista como un médico más del equipo con una agenda de trabajo diaria, trasladando así la consulta externa desde el hospital al centro de salud. Haría al mismo tiempo de consultor para sus colegas de familia, filtrando y atendiendo las consultas de especialidad de los mismos, y en todo momento asesorándoles en el seguimiento de pacientes que han sido dados de alta en el hospital. Con una población gallega de unos 2,7 millones de habitantes, serían necesarios 55 médicos internistas más en toda la comunidad. De este modo, el acortamiento de las listas de espera para consultas de especialidades y la eficacia resolutiva del binomio médico de familia-internista serían exponenciales. De este modo, la labor del internista en consultas externas hospitalarias debería sufrir un cambio importante. Al recibir el alta, los enfermos hospitalizados no volverían a consultas externas, siendo dirigidos a los médicos de familia correspondientes siempre con la posible asesoría del internista del centro de salud. Las consultas externas de nuestra especialidad dejarían paso a consultas de alta resolución (de pacientes enviados desde los centros de salud o del servicio de urgencias) y consultas de alta especialización (enfermedades infecciosas, enfermedades autoinmunes y/0 sistémicas...). Me viene a la memoria un informe presentado hace tiempo por el Dr. Alfonso Varela (internista del CHUO) relatando el secuestro que sufre el paciente una vez que entra en alguna de las consultas externas de cualquier especialidad: tiempos de espera larguísimos, estudios pedidos de lenta realización y creación de nuevas consultas para otros especialistas, en un círculo infernal que parece no terminar nunca. En estas nuevas consultas hospitalarias la espera sería muy corta al estar ayudado el internista por el especialista consultor y la priorización real de las pruebas y estudios necesarios.

En los años previos a mi jubilación, mi tarea asistencial se centró en un área geográfica provincial de unos 50.000 habitantes atendidos por 50 médicos de familia (la mitad en el centro de salud de la capital de provincia y el resto en los distintos centros del área asignada. Los pacientes vistos, una vez realizadas las pruebas analíticas, de imagen, endoscópicas, etc., se ponían en contacto conmigo vía telefónica y tras valorar los resultados en el programa informático del SERGAS (IANUS), en aproximadamente un 50\% de las ocasiones el paciente podía ser dado de alta sin necesidad de realizar otra consulta presencial (recibiendo un informe de alta en su domicilio en un intervalo de 48 horas). De este modo la agenda de trabajo se redujo a la mitad, pasando a ayudar a los compañeros de otras áreas geográficas. Si me hubieran concedido un editor de texto con reconocimiento de voz (como ya disponían de él los servicios de urgencias y radiología) el paciente hubiera regresado a su domicilio con las copias del informe de alta en el mismo día de su primera 0, a lo sumo, segunda visita. El inicio de las consultas de alta resolución tuvo lugar en Gandía (Valencia), lugar que visité en mis vacaciones, y ya entonces, para hacerlas eficaces, precisaban de un trato preferencial por los servicios de laboratorio, imagen...

Una labor fundamental del internista es la tarea de consultor para el resto de especialidades. De la misma forma que los otros consultores acuden a la llamada del colega, así nosotros deberíamos acudir a los reclamos de los compañeros lo más rápidamente posible. Las estadísticas indican que más de un $80 \%$ de las consultas requeridas provienen de los servicios de cirugía y traumatología y, en mucho menor porcentaje, de psiquiatría, neurología y dermatología. Y como ya han apuntado en distintos hospitales de España, lo más eficaz sería que la mayoría de los pacientes ingresados en estos servicios recibieran una asistencia compartida (especialistas e internistas) con nosotros.

\section{Cambio en la gestión}

El último cambio consiste, como ya comenté previamente, en transformar una empresa ruinosa, que es el hospital, en otra eficaz. Para ello el gerente debería ser totalmente independiente y apolítico, elegido por méritos propios, con un presupuesto libre, presupuesto al que debe rendir cuentas anualmente. El Director Médico, elegido democráticamente por los médicos del hospital, asesoraría al gerente. Los jefes de las distintas unidades asistenciales no serán nunca vitalicios y, asimismo, deberían ser elegidos por sus integrantes cada 4 u 8 años (sin menoscabo de la carrera profesional). He dicho "unidades asistenciales" porque los "servicios" comienzan a estar obsoletos como está ocurriendo ya en el Hospital Karokinska de Suecia, con áreas asistenciales multidisciplinares englobando diversos especialistas. Como ocurre en el Hospital Clínico de Barcelona, los médicos que lo desearan podrían hacer horas extraordinarias por las tardes en sus hospitales. Además, los médicos deberían elegir su labor asistencial en la sanidad pública o en la privada, pero nunca en las dos a la vez. En la gestión de la sanidad no hay que olvidarse de la participación ciudadana y las asociaciones de pacientes ya que éstos son el centro de toda la sanidad.

La figura del jefe de estudios debería equipararse a la de director médico con sus tareas fundamentales, docente e investigadora. Ambos detectarían los "cuellos de botella", tales como los que existen en el servicio de anatomía patológica y en el de radiología. En el primer servicio sus médicos deberían hacer guardias de tarde todos los días del año incluidos sábados y festivos. Y en el segundo servicio, además de eliminar los equipos obsoletos (tenemos la mayor obsolescencia de Europa) deberíamos aumentar en número de TAC, RMN, PET, ecógrafos y utilizarlos las 24 horas del día durante todo el año.

Vuelvo al principio. Se precisarían fundamentalmente tres cosas: aumento del presupuesto para medicina primaria, pacto de Estado y los cambios referidos en primaria y hospitalización. Doy por hecho que podemos hacerlos, queremos hacerlos y sabemos hacerlo.

En la relación médico paciente nada hay más eficaz que la relación directa, la comunicación telemática y, tal vez en el futuro, telepática. Y pese a la mejoría que aportarán los Big Data, la inteligencia artificial y los algoritmos (que ya están aquí para quedarse) la medicina seguirá siendo humana, y, creo, seguirá siendo un arte. 